\title{
ON THE EXPONENTIAL GROWTH OF SOLUTIONS TO NON-LINEAR HYPERBOLIC EQUATIONS
}

\author{
H. CHI, H. POORKARIMI, J. WIENER \\ Department of Mathematics \\ Pan American University \\ Edinburg, Texas, 78509, USA \\ and
}

\section{S.M. SHAH}

Department of Mathematics

University of Kentucky

Lexington, Kentucky 40506, USA

(Received September 9, 1987)

\begin{abstract}
Existence-uniqueness theorems are proved for continuous solutions of some classes of non-linear hyperbolic equations in bounded and unbounded regions. In case of unbounded region, certain conditions ensure that the solution cannot grow to infinity faster than exponentially.
\end{abstract}

KEY WORDS AND PHRASES. Non-linear Hyperbolic Equation, Integral Equation, Existence-Uniqueness, Equivalent Norm, Contraction, Exponential Growth.

1980 MATHEMATICS SUBJECT CLASSIFICATION CODES. 35A05, 35B05, $35 B 35,35 L 10$.

\section{INTRODUCTION.}

In this paper we study the existence of a unique solution to some non-linear partial differential equations of hyperbolic type. These equations appear in a mathematical model for the dynamics of gas absorption [1], and the main interest is to find solutions of exponential growth to a non-linear hyperbolic equation with characteristic data. It is possible to investigate such problems by the method of successive approximations, after reducing the differential equation to a Volterra integral equation in two variables. However, here we use the method of equivalent (weighted) norms, which considerably reduces the volume of computations. It should be noticed that in [1], an asymptotic investigation of corresponding linear equations has been conducted as $t \rightarrow \infty$. Periodic and almost-periodic solutions of a similar class of non-linear hyperbolic equations have been studied in [2]. The method of successive approximations has been applied in [3] and [4] to find bounded solutions of non-linear hyperbolic equations with time delay, which arise in control theory and in certain biomedical models.

We consider the equation

$u_{x t}(x, t)=F\left(x, t, u(x, t), u_{x}(x, t)\right)$,

and pose for (1) the following initial and boundary conditions: 


$$
\begin{array}{ll}
\mathrm{u}(0, t)=u_{0}(t) ; & 0 \leq t \leq T \\
\mathrm{u}(\mathrm{x}, 0)=\varphi(\mathrm{x}) ; & 0 \leq \mathrm{x} \leq l
\end{array}
$$

where $u_{0}(t)$ and $\varphi(x)$ are given functions in the domain $\Delta:[0, \ell] \times[0, T]$, and we are interested in existence-uniqueness to problem (1) $-(2)$.

Two norms $\|\mathbf{x}\|,\|\mathbf{x}\|_{*}$ on a Banach space are called equivalent if there exist two positive numbers $p$ and $q$ such that

$$
\mathrm{p}\|\mathbf{x}\| \leq\|\mathbf{x}\|_{*} \leq \mathrm{q}\|\mathbf{x}\| \text {. }
$$

For example, if the function $x(t)$ belongs to the space of continuous functions on $[0, T]$, it is easy to see that the norms

$$
\|\mathbf{x}\|=\max _{0 \leq t \leq T}|\mathbf{x}(t)|,
$$

and

$$
\|\mathbf{x}\|_{*}=\max _{0 \leq t \leq T} e^{-L_{1} t}|x(t)|, \quad L_{1}>0
$$

are equivalent. In order to prove the existence of a unique continuous solution to our problem, we use a norm similar to (3) and choose $L_{1}$ so that a certain integral operator becomes a contraction.

\section{MAIN RESULTS}

We prove our first result for equation (1) with the initial and boundary conditions (2) as follows.

THEOREM 1. Assume the hypotheses:

(i) The function $u_{0}(t)$ is continuously differentiable on $[0, T]$ and $\varphi(x)$ is continuously differentiable on $[0, \ell]$.

(ii) The function $F(x, t, u, v)$ is continuous in $\Delta \times x^{2}$ and satisfies the Lipschitz condition

$$
|F(x, t, u, v)-F(x, t, \bar{u}, \bar{v})| \leq L[|u-\bar{u}|+|v-\bar{v}|]
$$

for $u, v, \bar{u}, \bar{v} \in \mathscr{R}$ uniformly with respect to $x, t$. Then problem (1)-(2) has a unique continuous solution in $\Delta$. Proof. We change equation (1) to

$$
u(x, t)=u_{0}(t)+\varphi(x)-\varphi(0)+\int_{0}^{x} \int_{0}^{t} F\left(\xi, \eta, u(\xi, \eta), u_{\xi}(\xi, \eta)\right) d \eta d \xi
$$

and introduce the operator

$$
A w(x, t)=u_{0}(t)+\varphi(x)-\varphi(0)+\int_{0}^{x} \int_{0}^{t} F\left(\xi, \eta, w(\xi, \eta), w_{\xi}(\xi, \eta)\right) d \eta d \xi
$$

on the space $c^{1}(\Delta)$ of all functions $w(x, t)$ continuously differentiable in $\Delta$.

We define a weighted norm in $c^{1}(\Delta)$ by the formula:

$$
\|w\|_{*}=\max _{\Delta} e^{-L_{1} t}\left[|w(x, t)|+\left|w_{x}(x, t)\right|\right]
$$

where the constant $L_{1}>0$ will be chosen later. Since $u_{0}(t), \varphi(x)$, are continuously differentiable and $F(x, t, u, v)$ is a continuous 
function of its variables, operator $\left(4^{\prime}\right)$ maps $c^{1}(\Delta)$ into $c^{1}(\Delta)$. Now, we want to show that $A$ is a contraction on $C^{1}(\Delta)$. Consider the difference

$$
\begin{aligned}
A w(x, t) & -A \bar{w}(x, t)= \\
& \int_{0}^{x} \int_{0}^{t}\left[F\left(\xi, \eta, w(\xi, \eta), w_{\xi}(\xi, \eta)\right)-F\left(\xi, \eta, \bar{w}(\xi, \eta), \bar{w}_{\xi}(\xi, \eta)\right)\right] d \eta d \xi
\end{aligned}
$$

for $w, \bar{w} \in c^{1}(\Delta)$ and apply the Lipschitz condition, then $|A w(x, t)-A \bar{w}(x, t)| \leq L \int_{0}^{x} \int_{0}^{t}\left[|w(\xi, \eta)-\bar{w}(\xi, \eta)|+\left|w_{\xi}(\xi, \eta)-\bar{w}_{\xi}(\xi, \eta)\right|\right] d \eta d \xi$ Consider the derivative of $A w(x, t)$ and $A \bar{w}(x, t)$ with respect to $x$, then

$$
\begin{aligned}
& \left|(A w(x, t))_{x}-(A \bar{w}(x, t))_{x}\right| \\
& \leq \int_{0}^{t}\left|F\left(x, \eta, w(x, \eta), w_{x}(x, \eta)\right)-F\left(x, \eta, \bar{w}(x, \eta), \bar{w}_{x}(x, \eta)\right)\right| d \eta \\
& \leq L \int_{0}^{t}\left[|w(x, \eta)-\bar{w}(x, \eta)|+\left|w_{x}(x, \eta)-\bar{w}_{x}(x, \eta)\right|\right] d \eta
\end{aligned}
$$

From here,

$$
\begin{aligned}
& e^{-L_{1} t}\left[|A w(x, t)-A \bar{w}(x, t)|+\left|(A w(x, t))_{x}-(A \bar{w}(x, t))_{x}\right|\right] \\
& s L \int_{0}^{x} \int_{0}^{t} e^{-L_{1}(t-\eta)} e^{-L_{1} \eta}\left[|w(\xi, \eta)-\bar{w}(\xi, \eta)|+\left|w_{\xi}(\xi, \eta)-\bar{w}_{\xi}(\xi, \eta)\right|\right] d \eta d \xi \\
& \quad+L \int_{0}^{t} e^{-L_{1}(t-\eta)} e^{-L_{1} \eta}\left[|w(x, \eta)-\bar{w}(x, \eta)|+\left|w_{x}(x, \eta)-\bar{w}_{x}(x, \eta)\right|\right] d \eta \\
& S L\|w-\bar{w}\|_{*} \int_{0}^{x} \int_{0}^{t} e^{-L_{1}(t-\eta)} d \eta d \xi+L\|w-\bar{w}\|_{*} \int_{0}^{t-L_{1}(t-\eta)} d \eta \\
& S \quad L\left[\frac{l}{L_{1}}\|w-\bar{w}\|_{*}+\frac{1}{L_{1}}\|w-\bar{w}\|_{*}=\frac{L(l+1)}{L_{1}}\|w-\bar{w}\|_{*} \cdot\right.
\end{aligned}
$$

If we pick $L_{1}>L(\ell+1)$ and define $q=\frac{L(\ell+1)}{L_{1}}$, then

$$
\|A w-A \bar{w}\|_{*} \leq q\|w-\bar{w}\|_{*},
$$

with $0<q<1$. This shows that the operator $A$ is a contraction and proves the theorem.

The following proposition concerns the solution behaviour of an equation linear with respect to $u_{x}(x, t)$ in an unbounded region as $t \rightarrow \infty$. Although this result is generalized in Theorem 3 , its proof is given for instructive purposes. 
THEOREM 2. For equation

$$
u_{x t}(x, t)+a(x, t) u_{x}(x, t)=f(x, t, u(x, t)),
$$

and the initial and boundary conditions

$$
\begin{array}{ll}
\mathrm{u}(0, t)=\mathrm{u}_{0}(t) ; & 0 \leq t<\infty \\
\mathrm{u}(\mathrm{x}, 0)=\varphi(\mathrm{x}) ; & 0 \leq \mathrm{x} \leq l
\end{array}
$$

assume:

(i) $\quad a(x, t)$ is continuous in $\Omega:[0, l] \times[0, \infty)$ and satisfies the condition $a(x, t) \geq \mathrm{m}$, where $\mathrm{m}$ is a constant, the function $\varphi(x)$ is continuously differentiable on $[0, l]$.

(ii) The function $f(x, t, u)$ is continuous in $\Omega \times \mathscr{R}$ and satisfies the Lipschitz condition

$|f(x, t, u)-f(x, t, v)| \leq L|u-v|$

for $u, v \in R$, uniformly with respect to $x, t$; the function $f(x, t, 0)$ satisfies the inequality

$$
|f(x, t, 0)| \leq K_{1} e^{L_{1} t}
$$

where $(x, t) \in \Omega, K_{1}$ is a constant, and

$$
\mathrm{L}_{1}>\mathrm{Le}-\mathrm{m} \text {. }
$$

(iii) The function $u_{0}(t)$ is continuously differentiable on $[0, \infty)$ and satisfies

$$
\begin{gathered}
\left|u_{0}(t)\right| \leq K_{2} e^{L_{1} t} \\
\text { for } t \in[0, \infty), K_{2} \text { is constant, and } L_{1} \text { satisfies (8). }
\end{gathered}
$$

Then problem $(6)-(7)$ has a unique continuous solution $u(x, t)$ in $\Omega$ and

$$
\sup _{\Omega} e^{-L_{1} t}|u(x, t)|<\infty .
$$

Proof. First, transform equation (6) to

$$
\begin{aligned}
u(x, t) & =u_{0}(t)+\int_{0}^{x} \varphi(\xi) e^{-\int_{0}^{t} a(\xi, s) d s} d \xi \\
& +\int_{0}^{x} \int_{0}^{t} e^{-\int_{\eta}^{t} a(\xi, s) d s} f(\xi, \eta, u(\xi, \eta)) d \eta d \xi
\end{aligned}
$$

and introduce the operator

$$
\begin{aligned}
A w(x, t) & =u_{0}(t)+\int_{0}^{x} \varphi(\xi) e^{-\int_{0}^{t} a(\xi, s) d s} d \xi \\
& +\int_{0}^{x} \int_{0}^{t} e^{-\int_{\eta}^{t} a(\xi, s) d s} f(\xi, \eta, w(\xi, \eta)) d \eta d \xi
\end{aligned}
$$

on the space $B(\Omega)$ of all functions $w(x, t)$ continuous in $\Omega$, with the norm 


$$
\|w\|_{* *}=\sup _{\Omega} e^{-L_{1} t}|w(x, t)|, \quad L_{1}>0 .
$$

Now we prove that the operator (10) maps $B(\Omega)$ into $B(\Omega)$. Indeed,

$$
\begin{aligned}
A w(x, t) & =u_{0}(t)+\int_{0}^{x} \varphi(\xi) e^{-\int_{0}^{t} a(\xi, s) d x} d \xi \\
& +\int_{0}^{x} \int_{0}^{t} e^{-\int_{\eta}^{t} a(\xi, s) d x}[f(\xi, \eta, w(\xi, \eta))-f(\xi, \eta, 0)] d \eta d \xi \\
& +\int_{0}^{x} \int_{0}^{t} e^{-\int_{\eta}^{t} a(\xi, s) d s} f(\xi, \eta, 0) d \eta d \xi
\end{aligned}
$$

and from the hypotheses of the theorem, we can write

$$
|\varphi(x)| \leq k, \quad e^{-L_{1} t}|f(x, t, 0)| \leq K_{1}, e^{-L_{1} t}\left|u_{0}(t)\right| \leq K_{2} .
$$

Hence, by virtue of Lipschitz condition (L), we obtain

$$
\begin{aligned}
& e^{-L_{1} t}|A w(x, t)| \leq K_{2}+K l e^{-\left(L_{1}+m\right) t} \\
& \quad+L \int_{0}^{x} \int_{0}^{t} e^{-\left(m+L_{1}\right)(t-\eta)} e^{-L_{1} \eta}|w(\xi, \eta)| d \eta d \xi+\frac{K_{1} l}{m+L_{1}} .
\end{aligned}
$$

Taking into account (11), this implies

$$
e^{-L_{1} t}|A w(x, t)| \leq\left[K_{2}+k e+\frac{K_{1} l}{m+L_{1}}\right]+L\|w\|_{* *} \int_{0}^{x} \int_{0}^{t} e^{-\left(m+L_{1}\right)(t-\eta)} d \eta d \xi .
$$

Therefore,

$$
\|A w\|_{* *} \leq K_{2}+\left(K+\frac{K_{1}}{m+L_{1}}\right) l+\frac{L l}{m+L_{1}}\|w\|_{* *} .
$$

From here we see that if $\|\mathrm{w}\|_{* *}$ is bounded, then $\|\mathrm{Aw}\|_{* *}$ is bounded, which proves that $A$ maps the space $B(\Omega)$ into itself.

Now, we evaluate $A u$ - Av for $u, v \in B(\Omega)$,

$$
\|A u-A v\|_{* *} \leq \frac{L l}{m+L_{1}} \lim _{\rightarrow \infty}\left[1-e^{-\left(m+L_{1}\right) T}\right]\|u-v\|_{* *} .
$$

Since the above limit is 1 , one can write

$$
\|A u-A v\|_{* *} \leq \frac{L l}{m+L_{1}}\|u-v\|_{* *},
$$

which shows that $A$ is a contraction on $\Omega$ and proves that problem $(6)-(7)$ has a unique continuous solution in $\Omega$ which is bounded in the sense of norm (11).

THEOREM 3. Assume for problem (1)-(7) the following hypotheses:

(i) The function $u_{0}(t)$ is continuously differentiable and satisfies $\left|u_{0}(t)\right| \leq k_{2} e^{L_{1} t}$ for $t \in[0, \infty)$, where $k_{2}$ is a constant, and $\varphi(x)$ is continuously differentiable on $[0, \ell]$.

(ii) The function $F(x, t, u, v)$ is continuous in $\Omega \times x^{2}$ and satisfies Lipschitz condition (L), uniformly in $x, t$. 
(iii) The function $F(x, t, 0,0)$ satisfies

$$
|F(x, t, 0,0)| \leq k_{3} e^{L_{1} t} \text {, }
$$

$\mathrm{K}_{3}$ is a constant and $\mathrm{L}_{1}>\mathrm{L}(\ell+1)$, where $\mathrm{L}$ is Lipschitz constant.

Then problem (1) - (7) has a unique continuous solution $u(x, t)$ in $\Omega$ and

$$
\sup _{\Omega} e^{-L_{1} t}|u(x, t)|<\infty .
$$

Proof. We reduce (1) to (4) and introduce the operator (4') on the space $c^{1}(\Omega)$ of all functions $w(x, t)$ continuously differentiable in $\Omega$, with the norm

$$
\|w\|_{*}=\sup _{\Omega} e^{-L_{1} t}\left[|w(x, t)|+\left|w_{x}(x, t)\right|\right]
$$

First, we prove that the operator maps $c^{1}(\Omega)$ into $c^{1}(\Omega)$. Indeed,

$$
\begin{aligned}
A w(x, t)= & u_{0}(t)+\varphi(x)-\varphi(0)+\int_{0}^{x} \int_{0}^{t}\left[F\left(\xi, \eta, w(\xi, \eta), w_{\xi}(\xi, \eta)\right)-F(\xi, \eta, 0,0)\right] d \eta d \xi \\
& +\int_{0}^{x} \int_{0}^{t} F(\xi, \eta, 0,0) d \eta d \xi
\end{aligned}
$$

and

$$
\begin{aligned}
{[A w(x, t)]_{x}=} & \varphi^{\prime}(x)+\int_{0}^{t}\left[F\left(x, \eta, w(x, \eta), w_{x}(x, \eta)\right)-F(x, \eta, 0,0)\right] d \eta \\
& +\int_{0}^{t} F(x, \eta, 0,0) d \eta .
\end{aligned}
$$

Hence,

$$
\begin{aligned}
\mid A w(x, t) & +\left|[A w(x, t)]_{x}\right| s\left|u_{0}(t)\right|+|\varphi(x)-\varphi(0)|+\left|\varphi^{\prime}(x)\right| \\
& +L \int_{0}^{x} \int_{0}^{t}\left[|w(\xi, \eta)|+\left|w_{\xi}(\xi, \eta)\right|\right] d \eta d \xi+\int_{0}^{x} \int_{0}^{t}|F(\xi, \eta, 0,0)| d \eta d \xi \\
& +L \int_{0}^{t}\left[|w(x, t)|+\left|w_{X}(x, t)\right|\right] d \eta+\int_{0}^{t}|F(x, \eta, 0,0)| d \eta .
\end{aligned}
$$

Multiplying the previous expression by $e^{-L_{1} t}$, we have

$$
\begin{aligned}
& e^{-L_{1} t}\left[|A w(x, t)|+|A w(x, t)|_{x}\right] \leq e^{-L_{1} t}\left|u_{0}(t)\right|+e^{-L_{1} t}|\varphi(x)-\varphi(0)| \\
& +e^{-L_{1} t}\left|\varphi^{\prime}(x)\right|+L \int_{0}^{x} \int_{0}^{t} e^{-L_{1}(t-\eta)} e^{-L_{1} \eta}\left[|w(\xi, \eta)|+\left|w_{\xi}(\xi, \eta)\right|\right] d \eta d \xi \\
& +\int_{0}^{x} \int_{0}^{t} e^{-L_{1}(t-\eta)} e^{-L_{1} \eta}|F(\xi, \eta, 0,0)| \text { d } \eta d \xi
\end{aligned}
$$




$$
\begin{aligned}
& +\int_{0}^{t} e^{-L_{1}(t-\eta)} e^{-L_{1} \eta}\left[\left|w(x, \eta)+w_{x}(x, \eta)\right|\right] d \eta \\
& +\int_{0}^{t} e^{-L_{1}(t-\eta)} e^{-L_{1} \eta}|F(x, \eta, 0,0)| d \eta .
\end{aligned}
$$

If we let $e^{-L_{1} t}\left[|\varphi(x)-\varphi(0)|+\left|\varphi^{\prime}(x)\right|\right] \leq K_{1}$, and take into account

$$
\int_{0}^{t} e^{-L_{1}(t-\eta)} d \eta \leq \frac{1}{L_{1}} \text {, }
$$

then

$$
\begin{aligned}
e^{-L_{1} t}\left[|A(x, t)|+\left|[A w(x, t)]_{x}\right|\right] \leq & K_{2}+K_{1}+\frac{L l}{L_{1}}\|w\|_{*}+\frac{K_{3} l}{L_{1}} \\
& +\frac{L}{L_{1}}\|w\|_{*}+\frac{K_{3}}{L_{1}} .
\end{aligned}
$$

Therefore,

$$
\|A w(x, t)\|_{*} \leq K_{2}+K_{1}+\frac{K_{3}(l+1)}{L_{1}}+\frac{L(l+1)}{L_{1}}\|w\|_{*},
$$

which proves that the operator (4) maps $C^{1}(\Omega)$ into $C^{1}(\Omega)$, For the proof of contraction, we simply repeat the corresponding computations of Theorem 1 .

\section{REFERENCES}

1. TIKHONOV, A. N. and SAMARSKII, A. A. Equations of Mathematical Physics, Pergamon Press, New York, (1963).

2. CORDUNEANU, C. and POORKARIMI, H. Qualitative problems for some hyperbolic equations, in "Differential Equations" (Ian w. Knowles and Roger T. Lewis, Ed.), North-Holland, New York, (1985), 107-113.

3. SHAH, S. M., POORKARIMI, H. and WIENER, J. Bounded solutions of retarded nonlinear hyperbolic equations, to appear.

4. POORKARIMI, H. and WIENER, J. Bounded solutions of nonlinear hyperbolic equations with delay, proceedings of the VII International Conference on Non-linear Analysis (July 28-Aug 1, 1986, V. Lakshmikantham, Ed.), 471-478, to appear. 


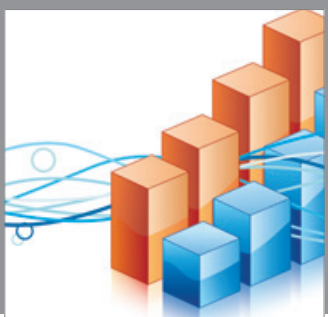

Advances in

Operations Research

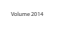

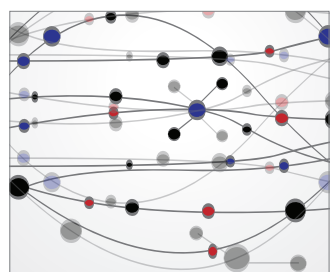

\section{The Scientific} World Journal
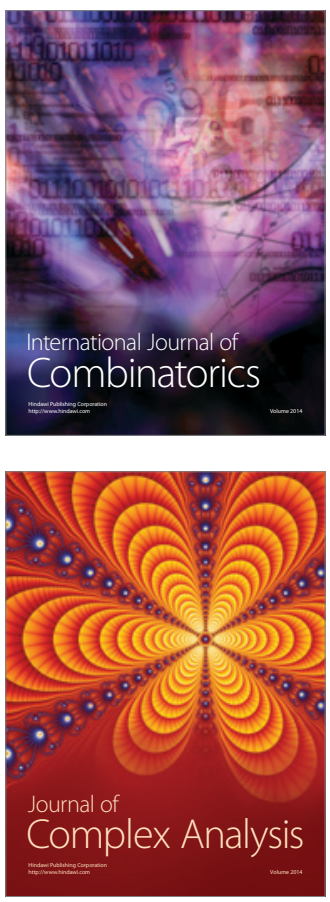

International Journal of

Mathematics and

Mathematical

Sciences
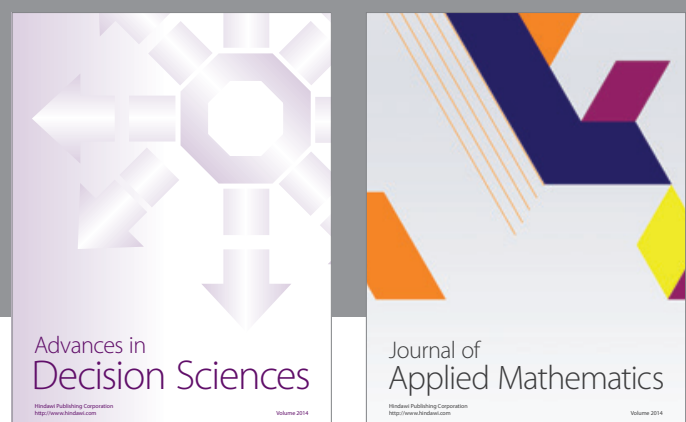

Journal of

Applied Mathematics
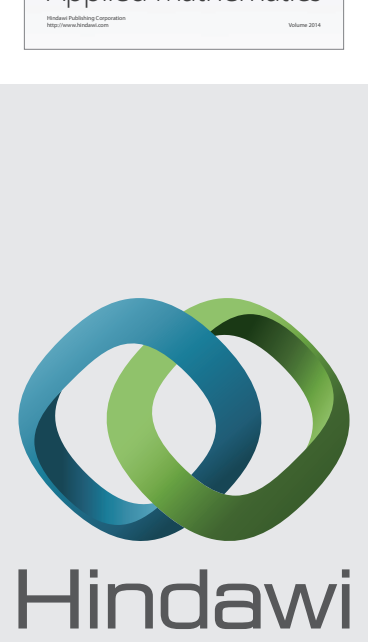

Submit your manuscripts at http://www.hindawi.com
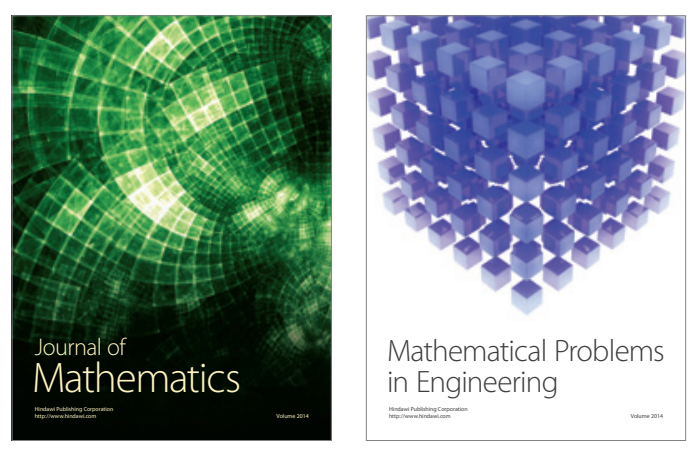

Mathematical Problems in Engineering
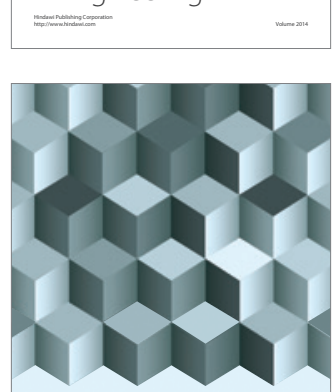

Journal of

Function Spaces
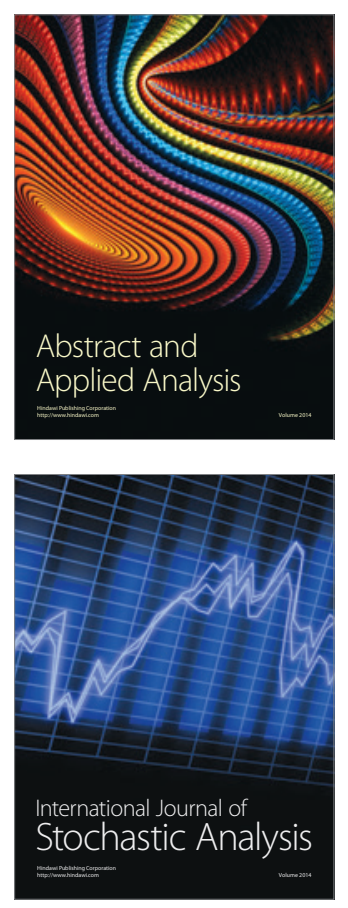

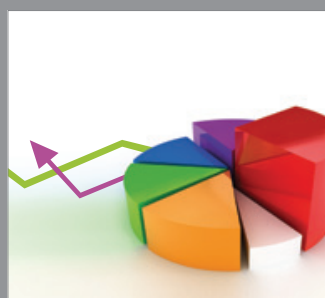

ournal of

Probability and Statistics

Promensencen
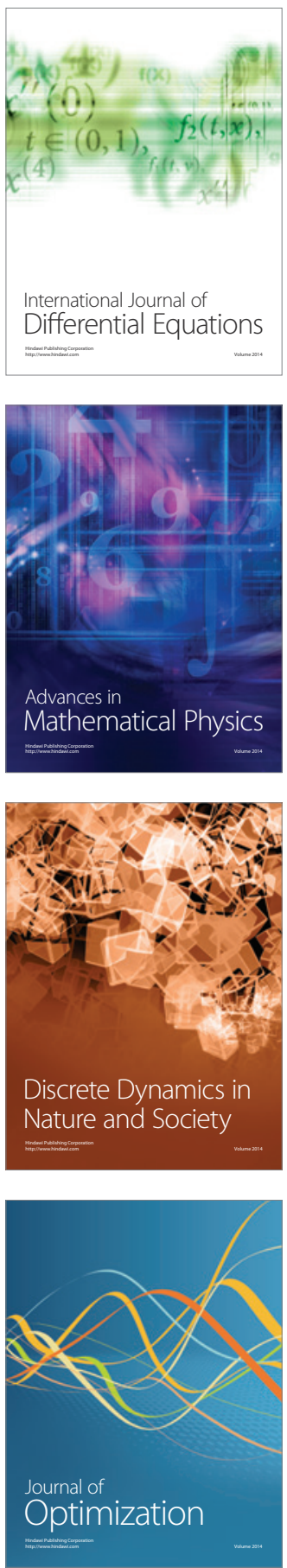